Address for Correspondence:

Dr. Muhammad Manzoorul Haque, Sindh Institute of Urology and Transplantation,

Karachi, Sindh, Pakistan.

Email: manzoorsiddiqui777@hotmail.com

\begin{tabular}{|l|}
\hline Access this article online \\
\hline $\begin{array}{l}\text { Website: } \\
\text { www.intern-med.com }\end{array}$ \\
\hline DOI: \\
10.2478/jtim-2018-0008 \\
\hline Quick Response Code: \\
\hline \\
\\
\\
\\
\\
\hline
\end{tabular}

\title{
Diagnostic accuracy of aspartate aminotransferase to platelet ratio index and fibrosis 4 scores in predicting advanced liver fibrosis in patients with end-stage renal disease and chronic viral hepatitis: Experience from Pakistan
}

\author{
Rajesh Kumar Wadhva ${ }^{1}$, Muhammad Manzoorul Haque ${ }^{1}$, Nasir Hassan Luck ${ }^{1}$, \\ Abbas Ali Tasneem ${ }^{1}$, Zaigham Abbas ${ }^{2}$, Muhammad Mubarak ${ }^{1}$ \\ 'Sindh Institute of Urology and Transplantation, Karachi, Pakistan; \\ 'Ziauddin Medical University Hospital, Karachi, Sindh, Pakistan
}

\begin{abstract}
Objectives: The aim was to assess the diagnostic accuracy of APRI and FIB-4 in assessing the stage of liver fibrosis in end stage renal disease (ESRD) patients with chronic viral hepatitis and to compare the two tests with standard tru-cut liver biopsy. Material and Methods: The study was conducted at Sindh Institute of Urology and Transplantation Karachi (SIUT) from May 2010 to May 2014. All ESRD patients, being considered as candidates for renal transplantation and in whom liver biopsy was performed were included. Fibrosis stage was assessed on liver biopsy using Ishak scoring system. The serum transaminases and platelet counts were used to calculate APRI and FIB-4 scores. Results: Out of 109 patients, hepatitis C and B virus infections were present in $104(95.4 \%)$ and $3(2.8 \%)$, respectively, while $2(1.8 \%)$ patients had both infections. The mean Ishak fibrosis score was $1.95 \pm 2$. Advanced fibrosis was noted in $37(34 \%)$ patients. Univariate analysis showed that advanced liver fibrosis was associated with lower platelets counts $(P=0.001)$ and higher aspartate aminotransferase (AST) $(P=0.001)$, alanine aminotransferase (ALT) $(P=0.022)$, APRI score $(P=0.001)$ and FIB-4 score $(P=0.001)$. On logistic regression analysis, only APRI score $(P<0.001)$ was found to be the independent variable associated with advanced liver fibrosis. APRI score cutoff $\geq 1$ indicating advanced fibrosis showed sensitivity, specificity, positive predictive value (PPV) and negative predictive value (NPV) of $91.9 \%, 90.3 \%, 82.9 \%, 95.6 \%$, respectively with area under the curve (AUC) of 0.97 . Similarly, a FIB-4 score cutoff $\geq 1.1$ had sensitivity, specificity, PPV and NPV of $70.27 \%$, $66.67 \%, 52 \%$ and $81.36 \%$, respectively with AUC of 0.74 . Conclusion: APRI is more accurate noninvasive test for assessing advanced liver fibrosis in ESRD patients as compared to FIB-4. It can be used to obviate the need for liver biopsy in this high risk population.
\end{abstract}

Key words: End-stage renal disease (ESRD), aspartate aminotransferase to platelet ratio index (APRI), fibrosis 4 scores(FIB-4), liver biopsy, liver fibrosis, platelet count

\section{INTRODUCTION}

In patients with end-stage renal disease (ESRD) and concomitant liver disease, secondary to chronic viral hepatitis, the assessment of liver fibrosis is essential when evaluating candidacy for kidney transplantation, as the presence of cirrhosis is usually considered a contraindication. [1] The liver biopsy specimen represents only $1 / 50,000$ part of the entire liver. The heterogeneity of liver fibrosis in viral infection and the inadequacy of sample size can cause considerable bias in the assessment of hepatic histology. ${ }^{[2-4]}$ Although widely performed and accepted as gold standard for diagnosing hepatic fibrosis, the liver biopsy is an invasive technique with associated morbidity. ${ }^{[5]}$ ESRD patients frequently have significant 
hemostatic disorders and hemorrhagic complications, posing additional risks for those undergoing this invasive procedures ${ }^{[6]}$ Hence, there is a need to develop accurate noninvasive tests to predict liver fibrosis, especially among dialysis dependent patients with viral infections like hepatitis $\mathrm{C}$ virus (HCV) and hepatitis $\mathrm{B}$ virus (HBV), in whom a higher risk for liver biopsy related complications has been observed. ${ }^{[7-8]}$ In the past few years, several biochemical indices have been proposed for the noninvasive evaluation of liver fibrosis in patients with chronic viral hepatitis and normal renal function. However, little data is available regarding this issue among the ESRD population. ${ }^{[9-11]}$ To minimize the need of liver biopsy and overcome its aforementioned limitations, there is a need to use tests that are less invasive and relatively easier to perform. We studied the diagnostic accuracy of two scoring systems, aspartate aminotransferase (AST) to platelet ratio index (APRI) and fibrosis 4 (Fib-4) score, which can be calculated from simple parameters obtained through blood tests in such situations.

\section{MATERIAL AND METHODS}

This cross-sectional study included consecutive adult ESRD patients with chronic viral hepatitis who were renal transplantation candidates and had undergone ultrasoundguided percutaneous liver biopsies in the Department of Hepatogastroenterology, at the Sindh Institute of Urology and Transplantation (SIUT) from May 2012 to May 2016. The data items were collected from medical records and liver biopsy reports. Patients with the following conditions were excluded: alcohol abusers (alcohol consumption exceeding $50 \mathrm{~g} /$ day), patients with clinical and radiological evidence of cirrhosis, hepatocellular carcinoma, patients with prior liver transplantation, prior interferon therapy, history of herbal medication and/or hepatotoxic drug use, immunosuppressive therapy, insufficient liver biopsy specimens for staging of fibrosis, and incomplete data on complete blood counts and liver panel tests. Written informed consent was taken from all patients. The study was approved by the ethical review committee (ERC) and was in accordance with Helsinki declaration 2013. All patients underwent ultrasound-guided percutaneous liver biopsies, using the 18-gauge Tru-Cut liver biopsy needle. The biopsies were reviewed by a single histopathologist who was unaware of the clinical and laboratory characteristics of the study subjects. Histological features were described using the Ishak scoring system. ${ }^{[12]}$ Advanced fibrosis was defined as an Ishak score of $5-6$, while a score of $0-4$ was considered as no to moderate fibrosis. Laboratory tests were performed one day prior to the liver biopsy. Serum aspartate aminotransferase (AST), alanine aminotransferase (ALT), prothrombin time (PT) including international normalized ratio (INR), and platelet counts were recorded. Serum AST and ALT were measured using UniCelDxC
800 Synchron clinical system and then expressed as times the upper limit of normal (ULN). The ULN for AST and ALT were taken as $40 \mathrm{U} / \mathrm{L}$ while the normal platelet count range was taken as 150,000 to $450,000 / \mathrm{mm}^{3}$. The APRI score was calculated according to the formula:APRI $=[($ AST $/$ ULN $) \times 100] /$ platelet count $10^{\circ} / \mathrm{L}$. The FIB-4 score was calculated according to the formula:FIB-4 = [Age (years) $\times$ AST $(\mathrm{IU} / \mathrm{L})] /\left[\right.$ Platelet count $\left(10^{9} / \mathrm{L}\right) \times$ ALT $\left.(\mathrm{IU} / \mathrm{L})^{1 / 2}\right] \cdot \cdot^{[3-15]}$ Ultrasound abdomen was performed by a senior radiologist and it was reviewed one day before the liver biopsy, and patients with spleen size of more than $13 \mathrm{~cm}$ were considered to have splenomegaly. ${ }^{[16]}$

\section{Statistics}

Statistical analysis was performed by SPSS software version 20.0 (SPSS Inc., Chicago, IL, USA). The following variables were included in the univariate analysis: demographics (age, sex), viral factors and other test results such as white cell count, platelet count, INR, serum total bilirubin, albumin, ALT, AST, and alkaline phosphatase (ALP). Categorical variables were analyzed by $\chi^{2}$ or Fisher's exact tests, while continuous variables with the Student's $t$ test. A 2-sided $P$ value of less than 0.05 was considered statistically significant. Univariate and stepwise multivariate analysis were used to identify variables associated with advanced fibrosis. The predictive accuracies of APRI and Fib-4 scores were tested by measuring the area under the receiver operating characteristics (AUROC). Based on the receiver operating characteristic (ROC) curve, the best cutoff points to predict the advanced fibrosis were chosen. Diagnostic accuracy was evaluated by calculating the sensitivity, specificity, and positive and negative predictive values (PPV and NPV).

\section{RESULTS}

\section{Patient Characteristics}

From May 2012 to May 2016, 109 hemodialysisdependent ESRD patients fulfilled the inclusion criteria and underwent percutaneous liver biopsies. The mean age was $33.3 \pm 9.4$ (range: $15-56$ ) years with a male predominance, $88(80.7 \%)$. Out of 109 patients, anti-HCV was positive in 104 (95.4\%), hepatitis B surface antigen (HBsAg) in 3(2.8\%), while 2(1.8\%) had HCV and HBV coinfection. Fifty-six (51\%) patients had received blood transfusions before starting dialysis. Of these patients, $13(11.9 \%)$ had no co-morbid condition while $83(76 \%)$ had hypertension, $9(8.3 \%)$ had diabetes mellitus and 4(3.7\%) had extra hepatic tuberculosis. All liver biopsy samples were considered adequate based on specimen size $(\geq 10 \mathrm{~mm})$ and number of portal tracts $\geq 5 / \mathrm{HPF}$. The mean biopsy size was $1.16 \pm 0.31 \mathrm{~cm}$ with a median of $1.01 \mathrm{~cm}$. The mean Ishak fibrosis score was $1.95 \pm 2$. No to moderate fibrosis ( 0 to 4 ) was observed in $72(66 \%)$ 
Wadhva et al: : Diagnostic accuracy of APRI and fibrosis 4 scores in predicting advanced liver fibrosis in patients with end-stage renal disease

\begin{tabular}{|c|c|c|c|}
\hline Variables (Mean \pm SD) & $\begin{array}{l}\text { No to moderate fibrosis } t \\
(n=72)\end{array}$ & $\begin{array}{l}\text { Advanced fibrosis } ¥ \\
(n=37)\end{array}$ & $P$ value \\
\hline Age (years) & $32.2 \pm 8.97$ & $35.4 \pm 9.97$ & 0.097 \\
\hline Platelets (X109/L) & 192.0686 & 128.76 & $<0.001$ \\
\hline Prothrombin time (s) & 11.14 & 11.34 & 0.311 \\
\hline INR & 1.0700 .13 & 1.09 & 0.474 \\
\hline Core size $(\mathrm{cm})$ & 1.175 & 1.178 & 0.956 \\
\hline Direct bilirubin (mg/dL) & 0.16 & 0.25 & 0.032 \\
\hline ALP (U/L) & 222.5 & 293.0 & 0.224 \\
\hline AST (U/L) & 34.0 & 113.3 & $<0.001$ \\
\hline ALT (U/L) & 43.3 & 73.29 & 0.022 \\
\hline GGT (U/L) & 78.2 & 128.3 & 0.065 \\
\hline Albumin $(\mathrm{g} / \mathrm{dL})$ & 3.26 & 3.28 & 0.796 \\
\hline APRI & 0.469 & 1.294 & $<0.001$ \\
\hline FIB- 4 & 0.96 & 2.06 & $<0.001$ \\
\hline
\end{tabular}

† Ishak Score 0-4; ¥lshak Score 5-6; ESRD: end-stage renal disease; ALP: Alkaline Phosphatase; AST:aspartate aminotransferase; ALT: alanine aminotransferase; GGT: gamma glutamyltransferase; APRI: AST to platelet ratio index; FIB-4: fibrosis 4.

and advanced fibrosis (5 to 6$)$ in $37(34 \%)$ patients. None of the included patients showed any clinical features of hepatic decompensation.

\section{Predictors of advanced fibrosis}

Univariate analysis of clinical and laboratory parameters in hemodialysis patients with and without advanced fibrosis was performed to determine the factors associated with extensive fibrosis (Table 1). The analysis revealed that lower platelet count $(P<0.001)$; higher AST $(P<0.001)$ and $\operatorname{ALT}(P=0.02)$; APRI score $\geq 1.0(P<0.001)$ and FIB-4 score $\geq 1.1(P<0.001)$ were associated with extensive fibrosis. Only the APRI and FIB-4 scores were employed in multivariate analysis, while other factors found to be significant in the univariate analysis like platelet count, AST and ALT were not included because APRI is dependent on platelet count and AST, while FIB-4 is dependent on ALT and platelet count. On logistic regression analysis, only APRI score $(P<0.001)$ was found to be the independent variable associated with significant liver fibrosis [odds ratio $(\mathrm{OR})=18.7,95 \%$ confidential interval $(\mathrm{CI})=6.1$ to 57.3$]$. In contrast, FIB-4 $(P=0.075)$ did not show such statistical significance $(\mathrm{OR}=3.49,95 \% \mathrm{CI}=0.88$ to 13.79$)$. Some parameters that did not show statistical significance, for example age, PT, INR, biopsy core length, ALP, gammaglutamyltransferase (GGT) and albumin.

\section{APRI and FIB-4 for predicting liver fibrosis}

From the univariate analysis, it was evident that increased AST and ALT levels and decreased platelet counts were significantly associated with liver fibrosis. As APRI score incorporated both AST and platelet count, it correlated
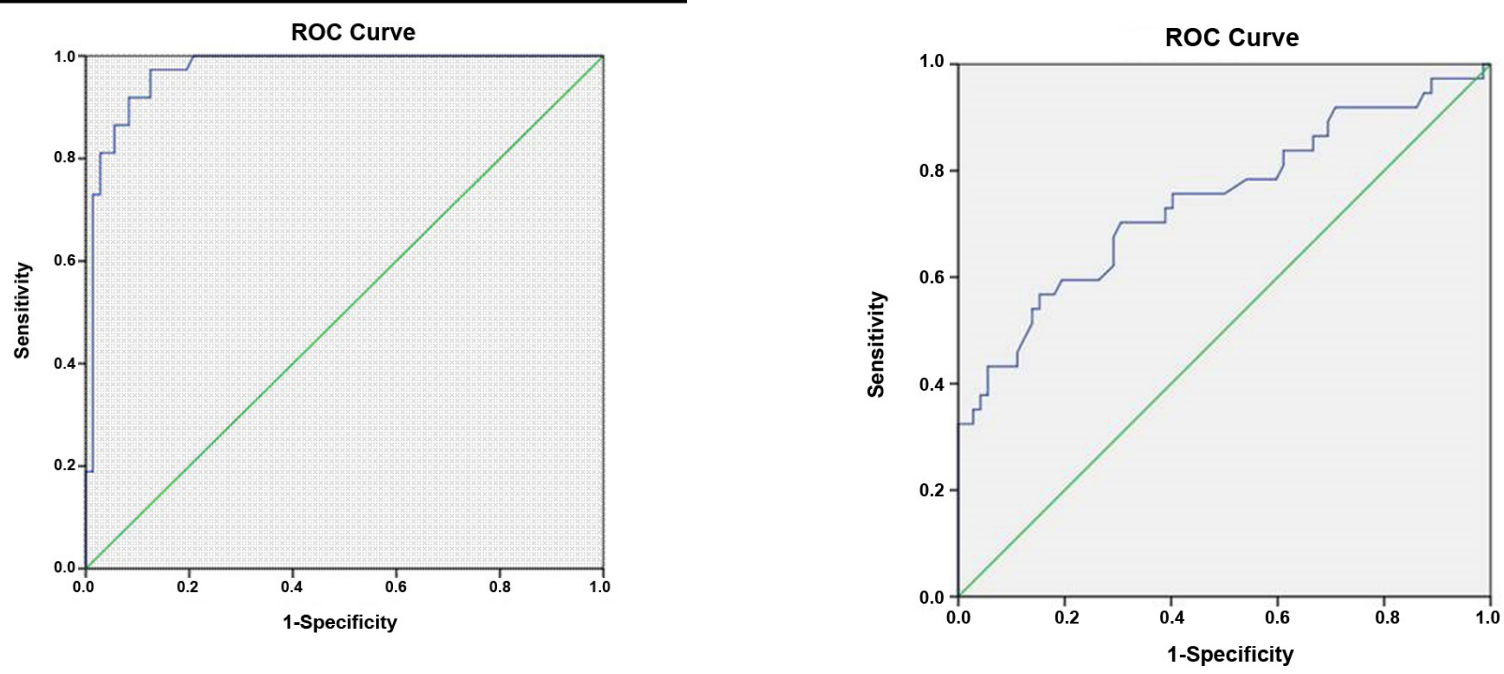

Figure 1: (a) ROC curve in the prediction of significant fibrosis (1a) APRI (AUROC=0.97); (b) ROC curve in the prediction of significant fibrosis FIB-4 (AUROC=0.74). 
better with liver fibrosis. ROC curve for APRI score was plotted to determine advanced fibrosis and the area under the curve (AUC) calculated was 0.97, as shown in Figure 1. The FIB-4 score was also found to be associated with advanced fibrosis $(P<0.001)$. ROC curve for FIB-4 plotted to determine advanced fibrosis revealed the AUC of 0.74 .

\section{Sensitivity and specificity of APRI and FIB-4 in predicting advanced fibrosis}

A cutoff point of APRI score of 1.0 was chosen to predict the presence or absence of advanced fibrosis. APRI score of $<1$ was considered as no to moderate fibrosis, while a score of $\geq 1$ was considered as extensive fibrosis. With this cutoff, the sensitivity, specificity, PPV and NPV of the test were found to be $91.9 \%, 90.3 \%, 82.9 \%$ and $95.6 \%$ respectively. Similarly, a cutoff point of 1.1 was chosen for FIB-4 to predict the presence or absence of extensive fibrosis. Considering the FIB-4 score of $<1.1$ as no to moderate fibrosis and FIB-4 scores of $\geq 1.1$ for extensive fibrosis, the sensitivity, specificity, PPV and NPV of the test were found to be $70.3 \%, 66.7 \%, 52 \%$ and $81.36 \%$ respectively.

\section{DISCUSSION}

The assessment of liver fibrosis in ESRD patients with chronic viral hepatitis is considered essential for indication of therapy, transplantation and prognosis. ${ }^{[17-19]}$ It is also important to rule out liver cirrhosis, which if present, constitutes a contraindication for lone kidney transplantation because of poor post-transplant survival. Liver biopsy is the gold standard method for staging and grading of liver disease, but it is an invasive procedure in which the frequency of pain at biopsy site is reportedly $20 \%$, while the incidence of mortality and other serious complications have been reported to be $0.01 \%$ and $0.3-0.57 \%$ respectively. ${ }^{[20-21]}$ On the other hand, liver biopsy represents only $1 / 50,000$ portion of the entire liver, due to which error of sampling is a likely possibility. To overcome this limitation, it is essential to use a thick needle (18-G) and collect 2 or more samples of sufficient length for correct assessment of fibrosis. Achieving this goal is difficult because of increased risk of bleeding among the hemodialysis patients who have impaired coagulation secondary to uremia, platelet dysfunction and heparininduced thrombocytopenia. ${ }^{[22-23]}$ After a liver biopsy, a stay in the hospital for 24-36 hours is required to observe for the development of any complications, which, if present, can prolong the stay and increase the overall cost of management. Due to these limitations of liver biopsy, the importance of noninvasive markers becomes more relevant in the assessment of liver fibrosis in patients with ESRD. Ideally, these markers should be simple, inexpensive, readily available, reliable and safe.
There are a number of noninvasive tests available to validate liver fibrosis. Among these, APRI and FIB-4 are frequently used for this purpose, although their utility in ESRD patients is not very well studied. In our study, both these markers were used to estimate the severity of liver fibrosis in patients with ESRD. The use of APRI and FIB-4 scores, which include AST and platelet count in predicting the presence or absence of advanced liver fibrosis, can be justified by the fact that in patients with advanced fibrosis, there is a decreased clearance of AST that is the result of increase hepatic fibrosis, reduced thrombopoietin release from hepatocytes and increased entrapment of platelets by the spleen. However, the use of heparin during hemodialysis may lead to thrombocytopenia and hence, affect the diagnostic accuracy of APRI and Fib-4 scores in predicting liver fibrosis in ESRD patients. This was one of the limitations of our study. Therefore, an adjustment of the cutoff values of APRI and FIB-4 scores is needed in this subset of patients for their validation. According to Bourliere et al., the noninvasive markers of fibrosis may have different diagnostic accuracies depending upon the prevalence of significant fibrosis in the studied population. ${ }^{[2]}$ This may explain the variability of results for APRI in different study subsets. In our study, APRI score accurately identified liver fibrosis in patients with ESRD with a sensitivity, specificity, PPV, NPV and an AUC of 0.97 for estimating Ishak fibrosis stage $>4$. The results derived from APRI score matched the liver biopsy findings in most of the cases. Therefore, it can be used to accurately predict advanced fibrosis, hence obviating the need of liver biopsy in this high-risk population. Also, because APRI is inexpensive, reproducible and readily available, it can replace the relatively more expensive and invasive methods of liver fibrosis assessment in resource-poor countries. We also evaluated the predictive utility of FIB-4 in our study. The FIB-4 score can easily be calculated through routine laboratory tests such as age, AST, ALT and platelet count. It was used to evaluate the presence of liver fibrosis in patients with HCV/ HIV co-infection in the APRICOT study. ${ }^{[25]}$ In our study, we found that FIB-4 score with cut off value $\geq 1.1$ had acceptable diagnostic accuracy. This is in contrast to previous studies performed in non-hemodialysis dependent patients, in which a cutoff value of $>1.45$ and $<3.25$ was used. ${ }^{[26-27]}$ Using a cutoff of $\geq 1.1$ in our study, we observed a high AUC value of 0.74 with high sensitivity (70.3\%), specificity $(66.7 \%)$, and NPV (81.36\%). This suggests that FIB-4 can also be used clinically to exclude advanced fibrosis in these patients.

When comparing the two noninvasive parameters, APRI score is more sensitive and specific test in predicting liver fibrosis, while FIB-4 score, although useful, is less accurate in this regard. Both these noninvasive tests are simple, reproducible, widely available and cost-effective when used 
to identify significant fibrosis in ESRD patients on renal replacement therapy. This study shows that APRI score can be used as a preliminary test to discriminate patients who need a liver biopsy from those who do not, in resource-poor settings where facilities for liver biopsy are scarce. Also, prompt referral for transplant evaluation of such patients can be achieved by indirectly reducing the number of the required liver biopsies.

\section{CONCLUSION}

APRI is a more sensitive, specific and accurate noninvasive test for assessing advanced liver fibrosis in ESRD patients with chronic viral hepatitis as compared to FIB-4. It can be effectively applied to identify advanced liver fibrosis and can obviate the need for liver biopsy, which is more invasive in this high-risk population. Because the APRI score is inexpensive and reproducible, it can be used to easily discriminate patients who need a liver biopsy from those who do not, before referring them for renal transplantation.

\section{DISCLOSURE}

There are no financial supports and potential competing interests to declare.

\section{REFERENCES}

1. Mathurin P, Mouquet C, Poynard T, Sylla C. Impact of hepatitis Band C virus on kidney transplantation outcome. Hepatology 1999;29:257-63.

2. Regev A, Berho M, Jeffers LJ, Milikowski C. Sampling error and intraobserver variation in liver biopsy in patients with chronic HCV infection. Am J Gastroenterol 2002;97:2614-8.

3. Siddique I, El-Naga HA, Madda JP, Memon A. Sampling variability on percutaneous liver biopsy in patients with chronic hepatitis $\mathrm{C}$ virus infection.Scand J Gastroenterol 2003;38:427-32.

4. Scheuer PJ. Liver biopsy size matters in chronic hepatitis: bigger is better. Hepatology 2003;38:1356-8.

5. Bravo AA, Sheth SG, Chopra S. Liver biopsy. N Engl J Med 2001;344:495500.

6. Sabovic M, Salobir B, PreloznikZupan I, Bratina P. The influence of the haemodialysis procedure on platelets, coagulation and fibrinolysis. Pathophysiol Haemost Thromb 2006;34:274-8.

7. Özdoğan M, Özgür O, Boyacıŏ̆lu S, Coşkun M, Kart H. Percutaneous liver biopsy complications in patients with chronic renal failure. Nephron 1996;74:442-3.

8. Terjung B, Lemnitzer I, Dumoulin FL, Effenberger W, Brackmann HH, Sauerbruch T, et al. Bleeding complications after percutaneous liver biopsy. Digestion 2003;67:138-45.

9. Varaut A, Fontaine H, Serpaggi J, Verkarre V, Vallet-Pichard A, Nalpas $\mathrm{B}$, et al. Diagnostic accuracy of the fibrotest in hemodialysis and renal transplant patients with chronic hepatitis $\mathrm{C}$ virus. Transplantation 2005;80:1550-5.
10. Furusyo N, Hayashi J, Kanamoto-Tanaka Y, Ariyama I, Etoh Y, Shigematsu M, et al. Liver damage in hemodialysis patients with hepatitis C virus viremia: a prospective 10-year study. Dig Dis Sci 2000;45:2221-8.

11. Boyacıoğlu S, Gür G, Yılmaz U, Korkmaz M, Demirhan B. Investigation of possible clinical and laboratory predictors of liver fibrosis in hemodialysis patients infected with hepatitis C virus. Transplant Proc 2004; 36: 50-2.

12. Ishak, Kamal, Baptista A, Bianchi L, Callea F, De Groote J, Gudat F, et al. Histological grading and staging of chronic hepatitis. J Hepatol 1995; 22: 696-9.

13. Ono E, Shiratori Y, Okudaira T, Imamura M, Teratani T. Platelet count reflects stage of chronic hepatitis C. Hepatol Res 1999;15:192-200.

14. Pohl A, Behling C, Oliver D, Kilani M, Monson P. Serum aminotransferase levels and platelet counts as predictors of degree of fibrosis in chronic hepatitis C virus infection. Am J Gastroenterol 2001;96:3142-6.

15. Wai CT, Greenson JK, Fontana RJ, Kalbfleisch JD, Marrero JA. A simple noninvasive index can predict both significant fibrosis and cirrhosis in patients with chronic hepatitis C. Hepatology 2003;38:518-26.

16. Dardenne AN. The spleen. In: Meire HB, Dewbury KCD, editors. Clinical Ultrasound. Edinburgh: Churchill Livingstone; 1993:pp.353-65.

17. Fabrizi F, Poordad FF, Martin P. Hepatitis C infection and the patient with end-stage renal disease. Hepatology 2002;36:3-10.

18. Fabrizi F, Dixit V, Messa P, Martin P. Interferon monotherapy of chronic hepatitis $\mathrm{C}$ in dialysis patients: meta-analysis of clinical trials. J Viral Hepat 2008;15:79-88.

19. Castera L, Pinzani M. Non-invasive assessment of liver fibrosis: are we ready? The Lancet 2010;375:1419-20.

20. Van der Poorten D, Kwok A, Lam T, Ridley L, Jones DB. Twenty-year audit of percutaneous liver biopsy in a major Australian teaching hospital. Intern Med J 2006;36:692-9.

21. Cadranel JF, Rufat P, Degos F. Practices of liver biopsy in France: results of a prospective nationwide survey. Hepatology 2000;32:477-81.

22. Vaziri ND, Toohey J, Paule P, Alikhani S, Hung E. Coagulation abnormalities in patients with end-stage renal disease treated with hemodialysis. Int J Artif Organs 1984;7:323-6.

23. King DJ, Kelton JG. Heparin-associated thrombocytopenia. Ann InternMed 1984;100:535-40.

24. Bourliere M, Penaranda G, Renou C, Botta-Fridlund D, Tran A. Validation and comparison of indexes for fibrosis and cirrhosis prediction in chronic hepatitis $\mathrm{C}$ patients: proposal for a pragmatic approach classification without liver biopsies. J Viral Hepat 2006;13:659-70.

25. Sterling RK, Lissen E, Clumeck N, Sola R, Correa MC. Development of a simple noninvasive index to predict significant fibrosis in patients with HIV/HCV coinfection. Hepatology 2006;43:1317-25.

26. Vallet-Pichard A, Mallet V, Nalpas B, Verkarre V, Nalpas A. FIB-4: An inexpensive and accurate marker of fibrosis in $\mathrm{HCV}$ infection: comparison with liver biopsy and fibrotest. Hepatology 2007;46:32-6.

27. Adler M, Gulbis B, Moreno C, Evrard S, Verset G. The predictive value of FIB-4 versus FibroTest, APRI, FibroIndex and Forns index to noninvasively estimate fibrosis in hepatitis $\mathrm{C}$ and nonhepatitis $\mathrm{C}$ liver diseases. Hepatology 2008;47:762-3.

How to cite this article: Wadhva RK, Haque MM, Luck NH, Tasneem AA, Abbas Z, Mubarak M. Diagnostic accuracy of aspartate aminotransferase to platelet ratio index and fibrosis 4 scores in predicting advanced liver fibrosis in patients with end-stage renal disease and chronic viral hepatitis: Experience from Pakistan. J Transl Intern Med 2018; 6: 38-42. 